\title{
Effects of Ginkgo biloba Extract EGb 761, Donepezil and their Combination on Central Cholinergic Function in Aged Rats
}

Christoph Stein, Julia Hopfeld, Helene Lau and Jochen Klein

Department of Pharmacology, College of Pharmacy, Goethe University Frankfurt, Germany.

Received, July 16, 2015; Revised, October 11, 2015; Accepted, October 26, 2015; Published, October 26, 2015.

\begin{abstract}
Purpose. Ginkgo extract EGb 761 and cholinesterase inhibitors have been shown to be effective in the treatment of dementia patients. In addition to neuroprotective effects, Ginkgo extract EGb 761 has been reported to elevate brain levels of certain neurotransmitters such as dopamine, noradrenaline, and acetylcholine. In the present study, we investigated the impact of EGb 761, donepezil and the combination of both drugs on the central cholinergic system in aged rats. Methods. 24 month old rats received EGb 761 (100 $\mathrm{mg} / \mathrm{kg} / \mathrm{day})$, donepezil $(1.5 \mathrm{mg} / \mathrm{kg} / \mathrm{day})$, the combination of both drugs or vehicle control by oral gavage for 14 days. We used microdialysis in rat hippocampus to monitor extracellular concentrations of acetylcholine (ACh), choline, glucose and lactate. Brain homogenates were prepared to measure activities of acetylcholinesterase (AChE), choline acetyltransferase (ChAT) and high affinity choline uptake (HACU). Results. While EGb 761 alone had no effect, donepezil and the combination of donepezil and EGb 761 increased basal ACh levels by 2- to 3-fold. Concomitantly, significant reductions of AChE and HACU were measured in both groups. No differences were seen between donepezil and the combination in these parameters. Treatment with EGb 761 decreased extracellular choline release and showed a tendency to moderately elevate ChAT activity. Conclusions. We found that donepezil and EGb 761 do not display a pharmacological interaction when given together. Adding EGb 761 did not modify the effects of donepezil on the hippocampal cholinergic system. Reduced choline levels indicate neuroprotective properties of EGb 761. Therefore, the combination of EGb 761 and donepezil may be beneficial in the treatment of Alzheimer's disease $(\mathrm{AD})$.
\end{abstract}

This article is open to POST-PUBLICATION REVIEW. Registered readers (see "For Readers") may comment by clicking on ABSTRACT on the issue's contents page.

\section{INTRODUCTION}

Alzheimer's disease (AD) is the most common adult-onset dementia, and its incidence highly correlates with age. The number of patients is projected to reach 115 million by 2050 (1). Effective treatments are needed to alleviate the great burden of the disease for patients, families and health care systems. According to the "cholinergic hypothesis", impairments in the central cholinergic system, especially in brain areas dealing with memory, learning, and emotional response are of great importance for the severity of clinical symptoms (2). Cholinergic therapy with acetylcholinesterase (AChE) inhibitors is a well-established strategy for symptomatic treatment of AD. Donepezil is a specific and reversible inhibitor of acetylcholinesterase (AChE) which increases acetylcholine (ACh) levels by reducing its breakdown. Donepezil is approved for mild-tomoderate AD in Europe and Japan, and for all AD stages in the USA and several other countries. Clinical studies have repeatedly shown improvements in cognitive functions, global assessment of change, behavioural symptoms and functional outcome measured by several scores such as the ADAS-cog (3-5).

Ginkgo biloba extract EGb 761 is approved in many European countries for the treatment of mild to moderate $\mathrm{AD}$, as well as for vascular and mixed dementia. Its efficacy and safety has recently been confirmed in three large, independent metaanalyses (6-8). Based on the clinical evidence, the German Institute for Quality and Efficacy in Health Care (IQWIG) and the European Medicines Agency (EMA) concluded that treatment with EGb 761 in a dose of $240 \mathrm{mg} / \mathrm{d}$ shows a beneficial effect on activities of daily living and an indication for improvement of cognition and quality of life (9). However, few data are available for the combination of EGb 761 and donepezil. One small randomised clinical trial compared the efficacy

Corresponding Author: Jochen Klein, Ph.D., Professor of Pharmacology and Clinical Pharmacy, Goethe University of Frankfurt, Biozentrum N260, Max-von-Laue-Str. 9, Frankfurt, Germany. E-mail: klein@em.uni-frankfurt.de 
of EGb 761 alone with that of donepezil alone and in combination with EGb 761 (10). The results revealed similar response rates in all groups with an apparent but not statistical significant tendency in favour of the combination measured by different scores for cognition, behaviour and related diseases. In a recent cohort study, beneficial effects on MMSE but not on ADAS-Cog from the combined therapy compared to cholinesterase inhibitor therapy alone were reported (11). Potential synergistic actions of the two drugs on the central cholinergic system were never investigated. This seemed interesting because $\mathrm{EGb}$ 761 has been shown to moderately increase extracellular levels of acetylcholine in the medial prefrontal cortex of freely moving rats (12). In the present study, we used microdialysis to study the impact of orally administered EGb 761, donepezil, and the combination of both on the hippocampal cholinergic system in aged rats. In addition to cholinergic markers, we also monitored extracellular levels of glucose and lactate, two major metabolites reflecting neuronal energy metabolism, and choline as a marker of neuronal death.

\section{METHODS}

\section{Animals}

Male Sprague-Dawley rats (24 month of age, 54 rats in total) were obtained from the Institute of Pharmacology and Toxicology of the Freie Universität Berlin. The animals were given two weeks to adapt to the new environment before experiments started. Rats were maintained on a 12 $\mathrm{h}$ light-dark cycle, at a room temperature of $22.5 \pm$ $2.5^{\circ} \mathrm{C}$ and a humidity of $50-70 \%$ with food and water available ad lib. The experimental procedures described here were carried out in accordance with the guidelines as set and approved by the responsible government agency (Regierungspräsidium Darmstadt, Germany).

\section{Treatment}

Four groups of rats received either vehicle solution, EGb 761, donepezil, or both drugs for four weeks. The first group (control; "Ctr") received an aqueous $30 \%$ sucrose solution (vehicle solution). The second group (EGb 761 group; "EGb") received $100 \mathrm{mg} / \mathrm{kg}$ EGb 761 extract suspended in vehicle solution. EGb $761^{\circledR}$, provided by Dr. Willmar Schwabe Co., Karlsruhe, Germany, is a dry extract from Ginkgo biloba leaves adjusted to $22-27 \%$ ginkgo flavonoids; 5$7 \%$ terpene lactones consisting of ginkgolides A, $\mathrm{B}$, and $\mathrm{C}$ and bilobalide; and less than $5 \mathrm{ppm}$ ginkgolic acids. The extract is stable when kept cold and dry, and solutions were prepared freshly for the present experiments. The third group (donepezil group; "Don") received $1.5 \mathrm{mg} / \mathrm{kg}$ donepezil dissolved in the sucrose solution. The fourth group (combination group; "EGb+Don") received $100 \mathrm{mg} / \mathrm{kg} \mathrm{EGb} 761$ extract and 1.5 $\mathrm{mg} / \mathrm{kg}$ donepezil. A dose of $100 \mathrm{mg} / \mathrm{kg} / \mathrm{d}$ for EGb 761 was chosen because it was reported to affect neurotransmitter levels in the brain in a previous study (12). Major constituents such as ginkgolides and bilobalide are known to enter the brain in relevant concentrations $(13,14)$. The relatively low dose of $1.5 \mathrm{mg} / \mathrm{kg} / \mathrm{d}$ donepezil was chosen because it is sufficient to decrease AChE activity in the brain (15) but not too high to mask potential effects of EGb 761 on the cholinergic system. Doses were given orally by gavage, once daily in the morning for four weeks. The last doses were given on the days of the experiments, i.e. immediately before the microdialysis experiment started.

\section{Implantation of the microdialysis probe}

On day 1, rats were anesthetised with isoflurane (Forene $^{\circledR}$, Abbvie, Ludwigshafen, Germany) in concentrations $(\mathrm{v} / \mathrm{v})$ of $4 \%$ isoflurane in air for induction and $1.5 \%$ isoflurane for maintenance of anesthesia by a vaporisator (Kent Scientific, USA). Self-made, I-shaped, concentric dialysis probes with an exchange length of $3 \mathrm{~mm}$ and a molecular cut-off of $10,000 \mathrm{Da}$ were constructed as previously described $(16,17)$. Using a stereotaxic frame (Stoelting, Chicago, USA), the probes were implanted into the right ventral hippocampus using the following coordinates (from bregma): AP: -5.2 $\mathrm{mm}$, L: $-5.2 \mathrm{~mm}$, and DV: $-7.0 \mathrm{~mm}$ (18). Glass ionomer eluting cement (Micron ${ }^{\circledR}$ i-Cem, PrevestDenPro, Heidelberg, Germany) was used to fix the probe on the skull. All animals received 2 $\mathrm{ml}$ of Ringer-lactate solution i.p. and were allowed to recover overnight.

\section{Microdialysis experiments}

Experiments were carried out in freely moving animals on the two days following probe implantation. Microdialysis probes were perfused with artificial cerebrospinal fluid (aCSF) containing $147 \mathrm{mM} \mathrm{NaCl}, 2.7 \mathrm{mM} \mathrm{KCl}, 1.2 \mathrm{mM}$ $\mathrm{MgCl}_{2}$ and $1.2 \mathrm{mM} \mathrm{CaCl}_{2}$ (all VWR, Darmstadt, Germany) at a perfusion rate of $2 \mu 1 / \mathrm{min}$. Samples were collected in intervals of $15 \mathrm{~min}$, immediately stored on ice and subsequently frozen at $-80{ }^{\circ} \mathrm{C}$ until analysis. After 90 min perfusion with aCSF, the dialysis fluid was switched to a CSF containing $0.1 \mu \mathrm{M}$ neostigmine (Sigma Aldrich, Munich, Germany); neostigmine was required to increase 
and stabilize ACh levels in vivo for the following behavioral experiments. After an additional 90 $\mathrm{min}$, the rats were placed in a novel environment ("open field") and microdialysis was continued $(17,19)$. The testing chamber was a gray plastic container $(45 \times 32 \times 20 \mathrm{~cm})$, and each rat was allowed to freely explore the chamber during the time of the experiment. Subsequently, the rats were returned to their home cage, and ACh efflux was monitored for another $90 \mathrm{~min}$. Animals were kept in their home cages overnight, with food and water available ad lib. On the second experimental day, baseline values (with neostigmine in the perfusion fluid) were again sampled for $90 \mathrm{~min}$, then the dialysis fluid was switched to aCSF containing 0.1 $\mu \mathrm{M}$ neostigmine and $1 \mu \mathrm{M}$ of scopolamine (Sigma Aldrich, Munich, Germany) while the rats remained in their home cage. After $90 \mathrm{~min}$, the perfusion fluid was switched back, and the rats were monitored for an additional $90 \mathrm{~min}$. After the end of the microdialysis experiments (approx. 8 hours after the last drug treatment), the rats were sacrificed and the left hemispheres were used to prepare homogenates for the measurement of acetylcholinesterase (AChE) activity, choline acetyltransferase (ChAT) activity and high-affinity choline uptake (HACU). The correct location of the microdialysis probes was verified by cutting 1 $\mathrm{mm}$ coronal sections of the right hemisphere.

\section{Measurement of acetylcholine, choline, glucose and lactate}

Acetylcholine and choline in dialysates were determined by microbore HPLC-ECD using the Eicom HTEC-500 system (Kyoto, Japan) that included degasser, low-speed pump, pre- and separation column, enzyme reactor carrying immobilised $\mathrm{AChE}$ and choline oxidase, and electrochemical detector with a platinum electrode operating at $500 \mathrm{mV}$ relative to the $\mathrm{Ag} / \mathrm{AgCl}$ reference electrode. The system is contained in a temperature-controlled cabinet. ACh was retained on the separation column and cleaved to choline and acetate by AChE; choline was then oxidised to hydrogen peroxide by choline oxidase. Hydrogen peroxide was detected electrochemically. The mobile phase consisted of $\mathrm{KHCO}_{3} 50 \mathrm{mmol} / 1$ (Merck, Darmstadt, Germany), EDTA-2Na 134.3 $\mathrm{mmol} / \mathrm{l}$ (BDH, Poole, UK) and sodium decane-1sulfonate $1.64 \mathrm{mmol} / 1$ (Alfa Aesar, Karlsruhe, Germany) in RotisolV ${ }^{\circledR}$ HPLC gradient grade water (Sigma-Aldrich, Munich, Germany), brought to $\mathrm{pH} 8.4$. The flow rate was $150 \mu \mathrm{l} / \mathrm{min}$. At an injection volume of $10 \mu$, the detection limit of this system was $2 \mathrm{fmol} /$ injection. Data acquisition was performed using EPC-500
PowerChrom $^{\circledR}$ software. Glucose and lactate concentrations in the dialysates were analysed by photometry using a CMA 600 microanalyser (CMA Microdialysis, Solna, Sweden).

\section{Acetylcholinesterase activity}

Brain homogenates were prepared by weighing the hemispheres and adding a 10-fold volume of isotonic HEPES-sucrose buffer (HEPES sodium salt 10mM, Sucrose 0.32 M, pH 7.4). Homogenization was performed with a tissue grinder (Potter S, B. Braun, Melsungen, Germany) at $1100 \mathrm{rpm}$ and 15 strokes. The homogenates were assessed for AChE activity using the Ellman method (20) with minor modifications $(21,22)$. Briefly, $50 \mathrm{ml}$ of tissue homogenate containing a final volume of $0.5 \%$ Triton $\mathrm{X}-100$ were centrifuged for $10 \mathrm{~min}$ at $12,000 \mathrm{~g}$ and $4^{\circ} \mathrm{C} .10 \mu \mathrm{l}$ of the supernatant was mixed with Ellman buffer and iso-OMPA (final concentration $100 \mu \mathrm{M}$ ). Acetylthiocholine and dithionitrobenzoic acid (1 $\mathrm{mM}$ and $500 \mu \mathrm{M}$ final concentrations, respectively) were added before measuring the absorbance at $405 \mathrm{~nm}$ using a Victor multilabel plate reader (Perkin Elmer, Bedford, USA). Enzyme activity was calculated using a standard curve prepared with each assay and expressed in relation to protein amount $(\mathrm{mU} / \mathrm{mg}$ protein). Protein determination was carried out using the Bradford method.

\section{Choline acetyltransferase activity}

ChAT was measured by a modification of the Fonnum method (23). Aliquots of brain homogenate $(0.5 \mathrm{mg} / \mathrm{ml}$ protein) were added to a total volume of $250 \mu \mathrm{l}$ reaction mix containing $0.5 \%$ Triton X-100, $0.3 \mathrm{M} \mathrm{NaCl}, 0.02 \mathrm{M}$ EDTA, $0.05 \mathrm{M} \mathrm{Na}_{3} \mathrm{PO}_{4}$ (pH 7.4), $2 \mathrm{mM}$ choline chloride, $1 \mathrm{mM}$ neostigmine bromide, and $0.5 \mathrm{mCi}$ $\left[{ }^{3} \mathrm{H}\right]$ acetylcoenzyme A. Following incubation at $37^{\circ} \mathrm{C}$ for $15 \mathrm{~min}$, the reaction was stopped by addition of $250 \mu$ lof ice-cold phosphate buffer (1 $\mathrm{mM}, \mathrm{pH}$ 7.4). ACh was extracted with $1 \mathrm{ml} 0.5 \%$ sodium tetraphenylboron in $85 \%$ toluene- $15 \%$ acetonitrile. After centrifugation, aliquots of $0.8 \mathrm{ml}$ were used for tritium quantification with a scintillation counter (Wallac system 1409, Perkin Elmer). ChAT activity was expressed as nmol of $\mathrm{ACh}$ formed per hour per $\mathrm{mg}$ of protein $(\mathrm{nmol} / \mathrm{h} / \mathrm{mg})$. Blank values were obtained by omitting the brain homogenate and subtracted.

\section{High affinity choline uptake}

HACU was determined in synaptosomes following a previously described, slightly modified protocol $(16,24,25)$. To obtain the P2 fraction, brain 
homogenates were centrifuged at $1000 \mathrm{~g}$ for 10 $\min$ and then at $17,000 \mathrm{~g}$ for $10 \mathrm{~min}$. The resulting pellets (containing the synaptosomes) were used for HACU determination. Aliquots were incubated at $30^{\circ} \mathrm{C}$ in the presence of $0.5 \mu \mathrm{M}\left[{ }^{3} \mathrm{H}\right]$ choline (diluted to $0.5 \mathrm{Ci} / \mathrm{mmol}$; Biotrend, Cologne, Germany) in Krebs-Henseleit buffer (KHB; containing $\mathrm{NaCl} 115 \mathrm{mM}, \mathrm{KCl} 7.1 \mathrm{mM}, \mathrm{CaCl}_{2} 1.2$ $\mathrm{mM}, \mathrm{MgSO}_{4} 1.2 \mathrm{mM}, \mathrm{NaHCO}_{3} 25 \mathrm{mM}, \mathrm{Na}_{2} \mathrm{HPO}_{4}$ $1.5 \mathrm{mM}$, glucose $12.8 \mathrm{mM}$, and saturated with carbogen adjusting to $\mathrm{pH}$ 7.2-7.4). Incubations were done both in the presence and absence of 1 $\mu \mathrm{M}$ hemicholinium-3 (HC-3). Choline uptake was stopped after $5 \mathrm{~min}$ by placing the reaction mix on ice and by washing with ice-cold KHB. After three centrifugation $(14,000 \mathrm{~g}, 10 \mathrm{~min})$ and washing (KHB buffer) steps, the pellets were solubilised in $4.5 \mathrm{ml}$ scintillation fluid (IRGA-SAFE PLUS, Perkin Elmer) and $0.5 \mathrm{ml}$ methanol and used for tritium quantification by liquid scintillation counting. The HC-3-sensitive, high-affinity choline uptake was calculated as the difference between uptake in the absence and presence of HC3 and expressed as dpm/mg protein. Protein determination was carried out using the Bradford method.

\section{DATA ANALYSIS AND STATISTICAL EVALUATION}

Concentrations of $\mathrm{ACh}$, choline, metabolites and enzyme activities were expressed as means \pm standard error of the mean (SEM) for the respective group with the number of experiments indicated in figure legends. Metabolites, HACU, AChE and ChAT activity were analyzed by oneway analysis of variance (ANOVA) with Bonferroni's post-tests, or with Student's t-test. $\mathrm{ACh}$ and choline time courses as obtained by microdialysis were analysed using two-way analysis of variance (ANOVA) for repeated measures and Bonferroni's post-hoc test using Prism 5 (GraphPad ${ }^{\circledR}$ Software, San Diego, USA). Significance of data was assumed when statements could be made with $95 \%$ confidence.

\section{RESULTS}

Effects of the different treatments on extracellular levels of $\mathrm{ACh}$

The microdialysis probe has access to the extracellular space; hence, measured values reflect extracellular concentrations. Fig. 1A shows ACh levels of the four treatment groups obtained from hippocampus under basal conditions, i.e. without neostigmine in the perfusion fluid. 30 minutes after drugs were given by oral gavage, six continuous samples were collected reflecting basal ACh values. Mean ACh levels of all time points were $4.51 \pm 0.26 \mathrm{fmol} / 10 \mu \mathrm{l}$ for controls, $13.7 \pm 0.70$ fmol $/ 10 \mu$ l for donepezil treatment, $4.73 \pm 0.17$ $\mathrm{fmol} / 10 \mu \mathrm{l}$ for EGb 761-treated rats and $10.21 \pm$ $0.20 \mathrm{fmol} / 10 \mu \mathrm{l}$ for rats which received both donepezil and EGb 761. Thus, the two groups receiving donepezil had significantly higher ACh levels than the other two groups which did not differ substantially. While the donepezil group had higher ACh levels than controls throughout the 90 min of measurement, the $\mathrm{EGb}+\mathrm{Don}$ group reached similar ACh levels after 75-90 min.

Preliminary experiments showed that behavioural stimulation such as the "open field" increases ACh levels under basal conditions; however, those values were too variable to render useful results. Therefore, we stabilized ACh levels with the addition of $0.1 \mu \mathrm{M}$ neostigmine to the perfusion fluid which increased $\mathrm{ACh}$ concentrations to approximately 70 to 77 fmol/10 $\mu 1$ in each group (Fig. 1B). ACh levels further increased after placing the rats into a novel environment ("open field"). Maximum increases were $142 \pm 17 \mathrm{fmol} / 10 \mu \mathrm{l}$ for controls, $144 \pm 20$ $\mathrm{fmol} / 10 \mu \mathrm{l}$ for $\mathrm{EGb}$ 761-treated rats, $162 \pm 20$ $\mathrm{fmol} / 10 \mu \mathrm{l}$ for donepezil-treated rats and $147 \pm 23$ fmol/10 $\mu$ for rats receiving both EGb 761 and donepezil. Thus, while all rats responded with elevated ACh levels, the differences between the groups were not significant. Returning the animals back to their home cages yielded a small initial increase of ACh followed by lower ACh concentrations which returned to basal levels after $90 \mathrm{~min}$ in all treatment groups.

On the second day of experiments, basal levels of ACh were similar as towards the end of day 1 (Fig. 1C). Upon infusion of scopolamine, hippocampal ACh levels increased 3 to 4 -fold in all groups (Fig. 1C). ACh concentrations reached a maximum after 30 to 45 min infusion with $363 \pm$ $52 \mathrm{fmol} / 10 \mu \mathrm{l}$ for controls, $291 \pm 61 \mathrm{fmol} / 10 \mu \mathrm{l}$ for donepezil-treated rats, $345 \pm 41 \mathrm{fmol} / 10 \mu \mathrm{l}$ for EGb 761-treated rats and $314 \pm 96 \mathrm{fmol} / 10 \mu \mathrm{l}$ for rats that received both drugs. ACh concentrations slightly decreased approximately $30 \mathrm{~min}$ after the end of the scopolamine infusion but remained elevated for more than $90 \mathrm{~min}$. 

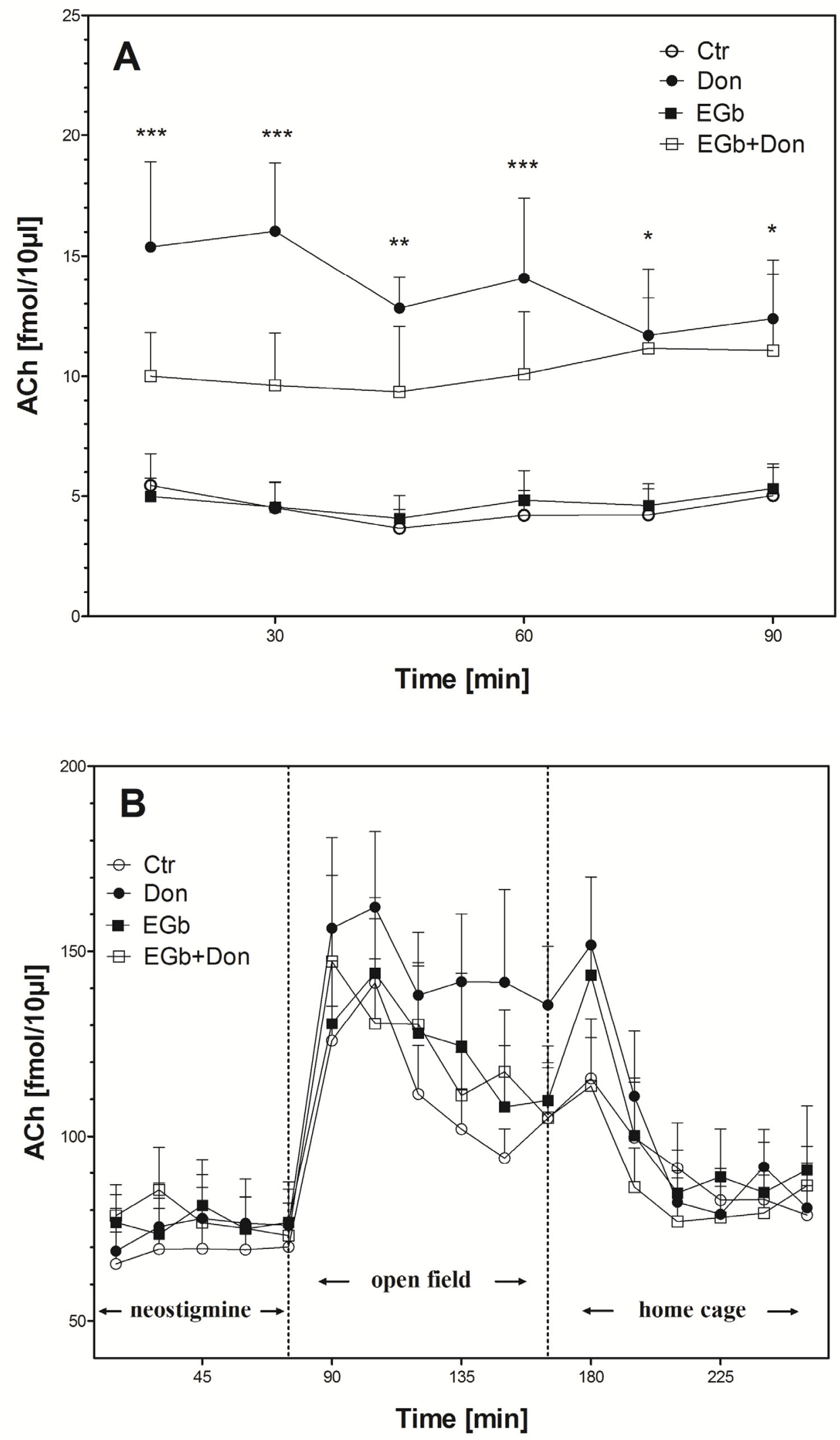
Figure 1 Continued.....

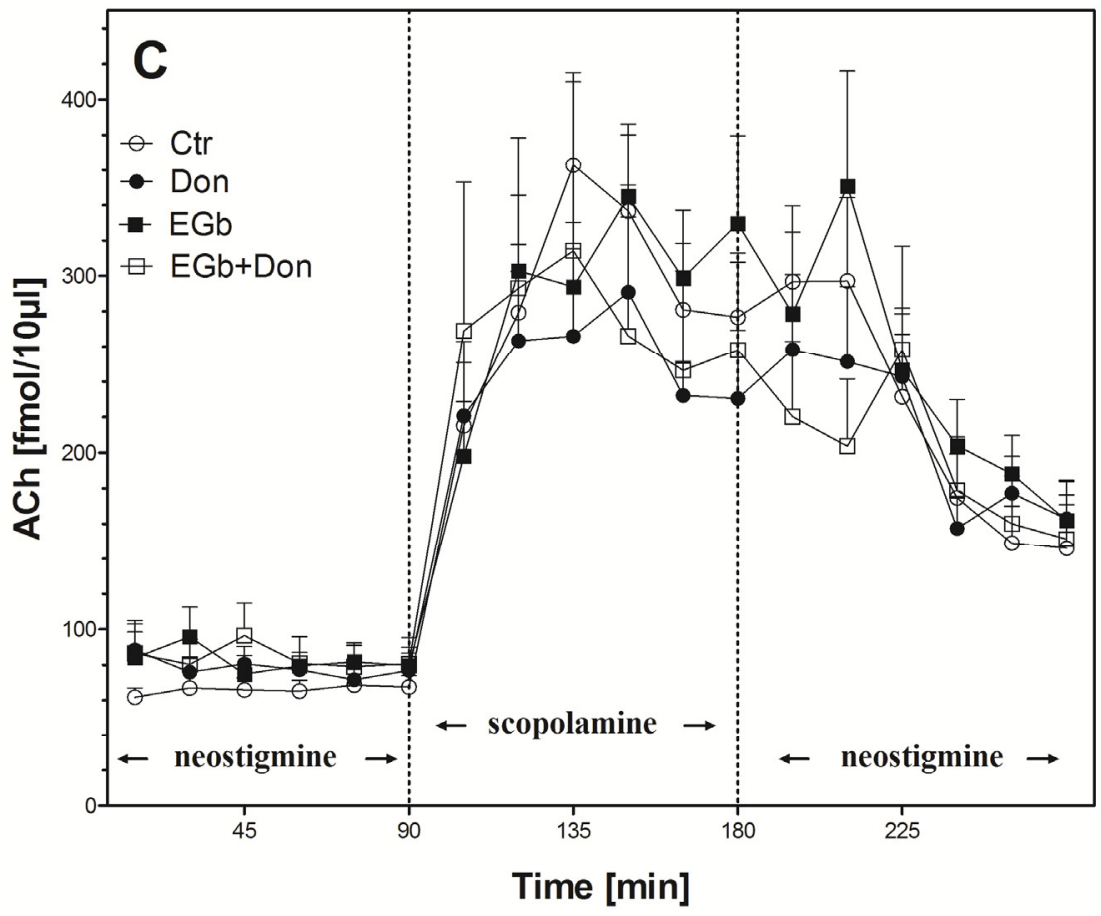

Figure 1. Extracellular concentrations of $\mathrm{ACh}$ in rat hippocampus sampled by microdialysis. Different groups of rats received treatments for 4 weeks (once per day) until immediately before start of microdialysis: control ("Ctr"; N=14); donepezil group ("Don") (1.5 mg/kg p.o.; N=12); EGb 761 group ("EGb") (100 mg/kg p.o.; N=14); combination group ("EGb+Don") (100 mg/kg and $1.5 \mathrm{mg} / \mathrm{kg}$ p.o.; $\mathrm{N}=14)$. Data is presented as means $\pm \mathrm{SEM}$ and is given as absolute values, not corrected for recovery. (A) Basal conditions without neostigmine. Statistical significance was calculated by two-way ANOVA for repeated measures (GraphPad Prism ${ }^{\circledR}$ ). Ctr vs. Don: $F_{1,75}=22.1, p<0.001$; Ctr vs. EGb: $F_{1,85}=0.02, p=$ 0.88; Ctr vs. $E G b+D o n, F_{1,80}=5.74, p=0.03$; $E G b$ vs. $E G b+D o n: F_{1,75}=5.43, p=0.03$; Don vs. EGb+Don: $F_{1,65}:=1.36$, $\mathrm{p}=0.26$. Bonferroni post test: $*, \mathrm{p}<0.05 ; * *, \mathrm{p}<0.01 ; * * *, \mathrm{p}<0.001$ vs. Ctr. (B) Behavioral activation on day 1 . Perfusion with aCSF containing $0.1 \mu \mathrm{M}$ neostigmine. 0 to $75 \mathrm{~min}$ basal values, 75 to 165 min behavioral activation (exposure to novel environment, "open field"), 165 to $255 \mathrm{~min}$ home cage. Statistical analysis (two-way ANOVA for repeated measures, GraphPad Prism ${ }^{\mathbb{R}}$ ): $\mathrm{F}_{3,943}=0.48 ; \mathrm{p}=0.69$. (C) Pharmacological activation on day 2. Perfusion with aCSF containing $0.1 \mu \mathrm{M}$ neostigmine (0-90 min), 90 to 180 min perfusion with aCSF containing $0.1 \mu \mathrm{M}$ neostigmine plus $1 \mu \mathrm{M}$ scopolamine, 180 to $270 \mathrm{~min}$ perfusion with aCSF containing $0.1 \mu \mathrm{M}$ neostigmine. Statistical analysis (twoway ANOVA for repeated measures; GraphPad Prism $\left.{ }^{\circledR}\right): F_{3,680}=0.18 ; p=0.91$.

\section{Cholinergic enzymes and transporters}

Cholinergic parameters were measured in brain homogenates of the left hemisphere. Ex vivo- data of the AChE activity (Fig. 2A) reflect the rate of breakdown of acetylcholine. Donepezil and EGb 761 plus donepezil significantly decreased AChE activity compared to control, whereas EGb 761 treatment alone did not affect AChE. Fig. 2B shows the effect of the treatments on ChAT activity, the cytosolic enzyme which catalyzes ACh synthesis. No differences could be seen between groups when tested by one-way ANOVA. However, ChAT activity in the EGb 761-treated group $(35 \pm 1.2 \mathrm{nmol} \mathrm{ACh} / \mathrm{h} / \mathrm{mg})$ seemed to be higher compared to control $(32 \pm 1.2 \mathrm{nmol}$ $\mathrm{ACh} / \mathrm{h} / \mathrm{mg} ; \mathrm{p}=0.09$ ). High affinity choline uptake (HACU; Fig. 2C), measured ex vivo, is a parameter for the in vivo-turnover of $\mathrm{ACh}$ prior to sacrifice (23). While there is an indication of donepezil reducing HACU $(p=0.07)$, treatment with the combination of donepezil plus EGb 761 resulted in a significant decrease of HACU activity. In contrast, HACU was not altered by EGb 761 treatment alone.

\section{Effects of drug treatments on extracellular levels of choline \\ Extracellular choline derives from breakdown of $\mathrm{ACh}$, but also from the hydrolysis of choline- containing phospholipids such as phosphatidylcholine (PC); PC is hydrolyzed by phospholipase $\mathrm{A}_{2}$ and $\mathrm{D}$, e.g. upon cellular damage (26).}



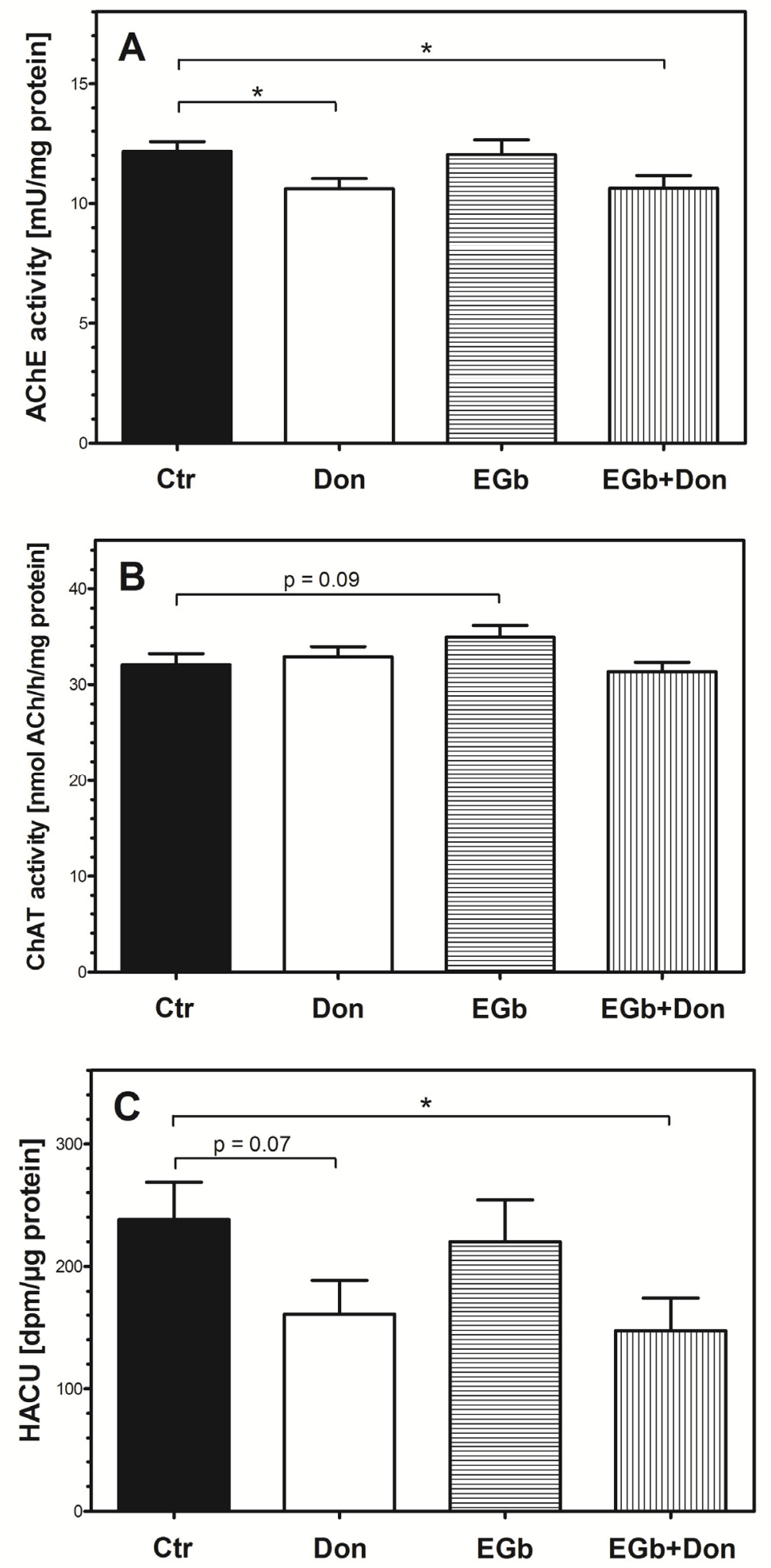

Figure 2. Effect of the drug treatments on the activity of cholinergic enzymes and transporters. (A) Acetylcholinesterase activity and (B) choline acetyltransferase activity were measured in the supernatant of brain homogenates whereas (C) high affinity choline uptake was measured in the $\mathrm{P} 2$ fraction. Homogenates and $\mathrm{P} 2$ fractions were prepared approximately 8 hours after the last drug administration and stored at $-80^{\circ} \mathrm{C}$ until measurement. Results are expressed as mean $\pm \mathrm{SEM}$ with $\mathrm{N}=12-14$ each. Statistical analysis (one-way ANOVA with Bonferroni post-test $\left(\operatorname{GraphPad} \operatorname{Prism}^{\circledR}\right):(\mathrm{A}) \mathrm{F}_{3,31}=$ 2.77; $p=0.05$. Student's t-test: Ctr vs. Don, $p=0.02 ; C$ tr vs. $E G b+D o n, p=0.04$. (B) $F_{3,47}=2.06 ; p=0.12$. Student's t-test: Ctr vs. EGb, $\mathrm{p}=0.09$. (C) $\mathrm{F}_{3,47}=2.26 ; \mathrm{p}=0.09$. Student's t-test: Ctr vs. $\mathrm{EGb}+\mathrm{Don}, \mathrm{p}=0.04$; Ctr vs. Don, $\mathrm{p}=0.07$. ${ }^{*}, \mathrm{p}<$ 0.05 . 
In our HPLC assay, choline in microdialysate samples was measured simultaneously with ACh (see Methods). Fig. 3 illustrates changes of choline levels over time in the four treatment groups on day 1 of experiments, i.e. during basal conditions, neostigmine infusion and stimulation with the "open field". Interestingly, the EGb 761-treated group shows significant lower choline levels than controls and donepezil-treated groups. Mean choline concentrations in dialysates were $0.54 \pm$ $0.02 \mu \mathrm{M}$ for controls, $0.58 \pm 0.07 \mu \mathrm{M}$ for the donepezil-treated rats, and $0.60 \pm 0.07 \mu \mathrm{M}$ for $\mathrm{EGb}$ 761 plus donepezil-treated rats, but only $0.45 \pm$ $0.03 \mu \mathrm{M}$ for EGb 761 -treated rats (Ctr vs. EGb, $\mathrm{p}=0.03)$.

\section{Extracellular levels of energy metabolites: glucose and lactate}

Glucose, the most important source of energy for the brain, is also a precursor of acetyl-CoA, and therefore required for $\mathrm{ACh}$ synthesis. In microdialysates, basal levels of glucose were 0.31 $\pm 0.02 \mathrm{mM}$ for controls, $0.34 \pm 0.04 \mathrm{mM}$ for donepezil-treated rats, $0.34 \pm 0.04 \mathrm{mM}$ for $\mathrm{EGb}$ 761-treated rats and $0.30 \pm 0.04 \mathrm{mM}$ for the $\mathrm{EGb}$ 761 plus donepezil-treated rats $(\mathrm{p}>0.3)$; accordingly, the different drug treatments did not have an effect on basal glucose levels (Fig. 4A; empty bars). Glucose levels were unchanged during behavioural or pharmacological stimulation (data not shown).

Fig. 4A (filled bars) shows lactate concentrations under basal conditions. Lactate levels overall were not different between treatment groups. Fig. 4B illustrates, however, that lactate concentrations were elevated when rats were placed in the "open field". The increases were found to be significant in all treatment groups (paired t-test), but the extent of lactate mobilization was very similar in all groups. Enhanced lactate release could not be seen during pharmacological stimulation with scopolamine (data not shown).

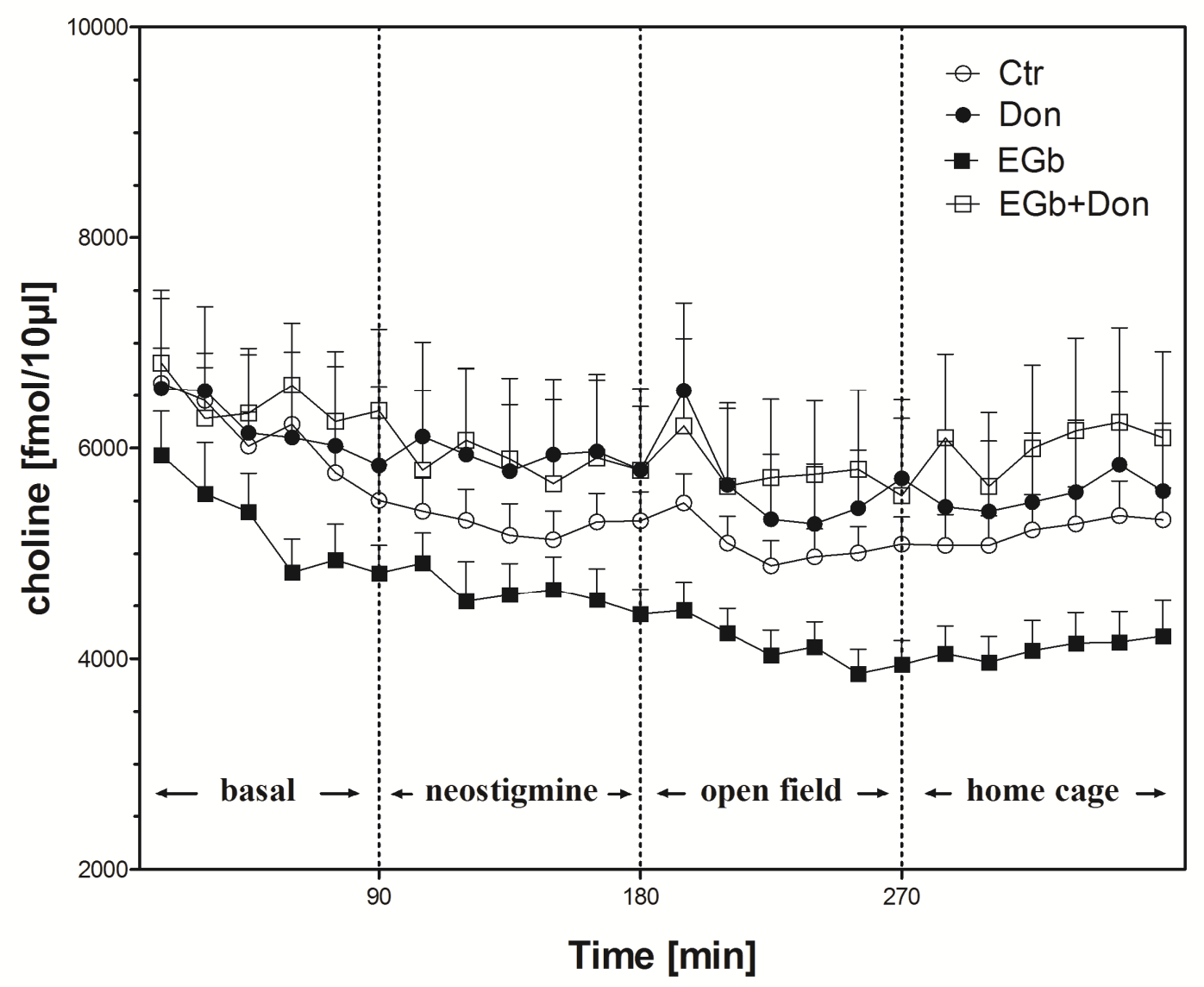

Figure 3. Extracellular concentrations of choline in rat hippocampus on day 1, sampled by microdialysis. Data were obtained using perfusion with aCSF (0-90 min, "basal"), with aCSF containing 0.1 $\mu$ M neostigmine (90-180 min), during behavioural activation (exposure to novel environment, "open field", from 180-270 min), and after return of rats to the home cage (270 to $360 \mathrm{~min})$. Results are means \pm SEM from the same samples as in Fig. 1A. Statistical analysis (twoway ANOVA for repeated measures, GraphPad Prism $\left.{ }^{\circledR}\right)$ : Ctr vs. Don, $F_{1,483}=0.43 ; p=0.52 ;$ Ctr vs. EGb, $F_{1,483}=5.5 ; p$ $=0.02 ;$ Ctr vs. $\mathrm{EGb}+$ Don, $\mathrm{F}_{1,506}=0.82 ; \mathrm{p}=0.37$. 

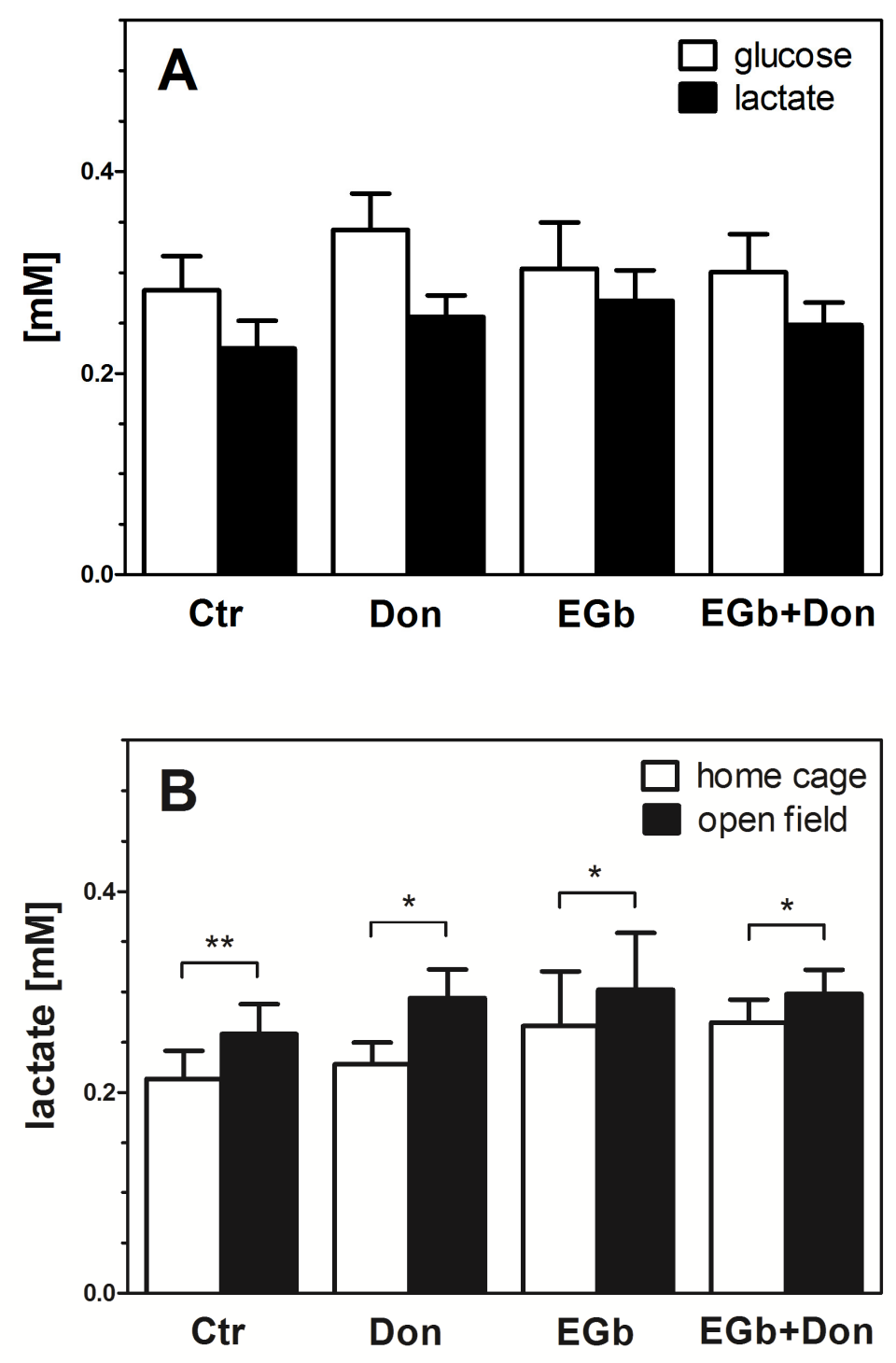

Figure 4. Energy metabolites under basal and stimulated conditions as sampled by microdialysis. (A) Extracellular glucose and lactate concentrations during basal conditions. (B) Effects of behavioural stimulation ("open field") on lactate levels. Concentrations were calculated as means of the first three samples. (A) Statistical analysis (one-way ANOVA with Bonferroni post-tests, $\left.G r a p h P a d \operatorname{Prism}^{\circledR}\right)$ : for glucose, $F_{3,31}=0.32 ; p=0.81$; for lactate, $F_{3,30}=0.56 ; p=0,64$ $(\mathrm{N}=8-10$ in each group). Student's t-test: Ctr lactate vs. EGb lactate, $\mathrm{p}=0.26$ (B) Statistical analysis (paired t-test, GraphPad Prism $\left.^{\mathbb{R}}\right)$ : Ctr, $\mathrm{p}<0.01$; Don, $\mathrm{p}=0.03 ; \mathrm{EGb}, \mathrm{p}=0.03 ; \mathrm{EGb}+\mathrm{Don}, \mathrm{p}=0.04(\mathrm{~N}=8-10$ in each group). *, $\mathrm{p}<0.05 ; * *$, $\mathrm{p}<0.01$.

\section{DISCUSSION}

The purpose of the present study was to examine the effects of single and combined treatments of donepezil, an AChE inhibitor, and EGb 761, an extract of Ginkgo biloba, on the cholinergic system. As outlined in the introduction, both drugs were shown to be effective for senile dementia and are therapeutically used in many countries; however, studies on their potential interaction are scarce. To increase the translational value of our study, we used moderate doses of the two drugs, applied orally, and we used aged rats as an animal model of the aging human.

The cholinergic system is an important target for senile dementia because its decline correlates with the severity of Alzheimer's disease (2). Increases of cholinergic transmission, albeit of moderate effectiveness, are the mainstay of current therapy with AChE inhibitors. In the present study, 
we applied the microdialysis technique, coupled to a highly sensitive HPLC system, to determine extracellular levels of ACh in the hippocampus. We chose this brain region because the hippocampushas a prominent role in cognitive processes and shows early dysfunction in dementia. Microdialysis is an ideal method to evaluate cholinergic function in vivo because sampling of $\mathrm{ACh}$ in the extracellular fluid reflects the activity of central cholinergic fibers. This is illustrated by the effect of AChE inhibition with donepezil which resulted in a significant increase of basal ACh concentrations after p.o. administration of the compound $(1.5 \mathrm{mg} / \mathrm{kg}$, Fig. 1A). The difference between the treatment groups disappeared when neostigmine was added to the perfusion fluid. Neostigmine was added to increase and stabilize ACh levels in vivo for the following experiments, and it has been shown previously that a low concentration of neostigmine $(100 \mathrm{nM}$ as in our study) does not impair the cholinergic response to behavioral or pharmacological stimulation (27; Stein, unpublished observations). We cannot exclude, however, that the addition of neostigmine masked a small effect of the treatments on ACh levels.

Exploration of a novel environment is known to enhance hippocampal ACh release in mice (16) and rats $(28,29)$, and we used this paradigm to monitor hippocampal cholinergic activity in aged rats. In our hands, all treatment groups responded with an approximately 2-fold increase of ACh release which subsided after return of the animals to the home cage (Fig. 1B). This response was slightly lower than the 3-fold increases observed before with young rats $(28,29)$, likely due to the old age of our animals and the loss of neuronal cells and dendritic spines taking place especially in the hippocampal region of aged rats $(30,31)$. On day 2 , increases in $\mathrm{ACh}$ levels were induced by pharmacological stimulation, i.e. infusion of scopolamine, which increases $\mathrm{ACh}$ release in vivo by blockade of inhibitory presynaptic receptors (19). Again, this effect requires partial blockade of AChE by neostigmine to activate the negative feedback mechanism. Importantly, all groups responded with an increase of $\mathrm{ACh}$ release approximately 3-fold indicating the functional preservation of cholinergic function. As with behavioral activation, the treated groups responded similarly to scopolamine.

The effect of donepezil was also seen when cholinergic parameters were measured ex vivo. As expected, AChE activity was decreased after donepezil. The moderate effect of the drug is explained by the low dose given and by the fact that approximately 8 hours passed between the last drug administration and sacrifice. An earlier study found a higher extent of AChE inhibition immediately following donepezil administration (15), but the half-life of donepezil in rats is only 3.6 hours (32), and a less prominent inhibition is, therefore, plausible after 8 hours. ChAT activity was unchanged after donepezil, but HACU was decreased. HACU activity is controlled by the presence of choline transporter CHT-1 in the plasma membrane which is internalized depending on the neuronal firing rate (33). The decrease of HACU activity after donepezil is plausible because an increase of ACh by AChE inhibition is expected to decrease ACh turnover due to the above mentioned, presynaptic muscarinic receptors which limit ACh release (19). A similar result was previously observed with galantamine, another $\mathrm{AChE}$ inhibitor (34). In contrast to the effects of donepezil on cholinergic parameters, the levels of choline, glucose and lactate were not affected by the drug.

EGb 761 is an extract containing ginkgolides, bilobalide and flavonoids with biological activity. Its mechanism of action in senile dementia is thought to be based on multiple mechanisms including neuroprotective effects (35); for instance, transgenic mice (36) and aged rats $(37,38)$ chronically treated with EGb 761 displayed improved spatial learning, memory and synaptic plasticity. Recent animal studies also suggested an effect of EGb 761 on neurotransmitter levels. A 14-day treatment with EGb 761 (100 or $300 \mathrm{mg} / \mathrm{kg}$, p.o.) increased dopamine and noradrenaline levels in the rat prefrontal cortex (PFC) (39). The present study which tested potential effects of EGb 761 on ACh levels was stimulated by the observation that $\mathrm{ACh}$ levels were slightly increased in frontal cortex following the administration of two flavonoids, quercetin glycoside and kaempferol glycoside (10 $\mathrm{mg} / \mathrm{kg}$ each) (12). However, in the present study oral treatment with $100 \mathrm{mg} / \mathrm{kg} /$ day $\mathrm{EGb} 761$ showed no effects on ACh levels in rat hippocampus; neither basal ACh levels, nor stimulated ACh levels were affected by EGb 761 (Fig. 1). The discrepancy to the earlier study (12) may be explained by measurements in another brain region. Moreover, the present study used a moderate dose $(100 \mathrm{mg} / \mathrm{kg} / \mathrm{d}$ p.o. $)$ of extract which contains only about $4.5 \%$ of quercetin and kaempferol, respectively.

EGb 761 treatment also did not change AChE and HACU activity, further illustrating that the extract had no direct effect on ACh levels in our study. However, two findings indicated a possible 
neuroprotective effect of the extract. First, rats treated with EGb 761 had significantly lower choline levels than the other groups. It is important to note that the extracellular levels of choline are poorly correlated with ACh release and breakdown, a fact that is also obvious in our study because addition of neostigmine or scopolamine, or exposure to the open field did not affect choline levels (Fig. 3). Instead, extracellular levels of choline are known to be dependent on the metabolism of choline-containing phospholipids. Under a variety of pathological conditions including ischemia and excitotoxicity, choline levels increase due to phospholipase $\mathrm{A}_{2}$ activation and hydrolysis of phosphatidylcholine (26). Ginkgo extract EGb 761 is known from our previous studies to strongly inhibit membrane phosphatidylcholine breakdown and glutamate release and to stabilize mitochondrial functions $(40,41,42)$. Interestingly, the effect of EGb 761 on choline was inhibited by donepezil (Fig. 3), probably because the increase of (excitatory) cholinergic transmission induced by donepezil counteracted the choline-lowering effect of EGb 761.

Measurement of cholinergic enzymes suggested a moderate increase of ChAT activity by EGb 761 treatment. ChAT, the enzyme which is responsible for the synthesis of $\mathrm{ACh}$, has been shown to decrease with progression of dementia (43), and a stabilization of ChAT activity may contribute to improved cognitive function. Although the effects of EGb 761 on ChAT activity did not reach significance, they are in agreement with a neuroprotective and activity-enhancing effect of the extract which was previously described in other studies (35).

While cholinergic parameters responded to drug treatments in this study, the levels of glucose, the major energy source of the brain, was not affected in rat hippocampus. Similarly, lactate levels did not respond to drug treatment. Interestingly, lactate levels increased upon exposure of rats to the open field in all four treatment groups (Fig. 4B). These increased lactate concentrations, earlier thought to reflect ischemic conditions, are now recognized to reflect neuronal activation (44). Astrocyte-derived lactate in the extracellular space is known to increase during physiological stimulation (19) and to support neuronal function, especially during high neuronal activity (44).

In summary, donepezil and EGb 761 are two drugs with different mechanisms of action that are beneficial in senile dementia. The present study tested co-administration of these two drugs in aged rats in an attempt to elucidate potential interactions. However, while effects were noted on several parameters, we did not detect major and significant interactions between the two drugs. In other words, while EGb 761 has no direct effects on ACh levels, it did also not interfere with the well-known cholinergic properties of donepezil. Donepezil is a well-established drug for the symptomatic treatment of $\mathrm{AD}$. It improves cognition, behavioral symptoms and quality of life by increasing levels of ACh in the brain. Our study shows that EGb 761 has no direct effects on ACh levels but is beneficial by its neuroprotective action. We conclude that EGb 761, while it does not directly affect the central cholinergic system, evidently does not interfere with the cholinergic effects of donepezil. As a consequence, a combination of both drugs might yield additive effects and should be further explored clinically.

\section{ACKNOWLEDGEMENT}

The study was supported by Dr. Willmar-Schwabe GmbH \& Co. KG, Karlsruhe, Germany.

\section{REFERENCES}

1. Prince M, Jackson J. World Alzheimer Report 2009. Alzheimer's Dis Int. 2009;1-96.

2. Schliebs R, Arendt T. The significance of the cholinergic system in the brain during aging and in Alzheimers's disease. J Neural Transm. 2006;113:1625-44.

3. Feldman H, Gauthier S, Hecker J, Vellas B, Subbiah P, Whalen E. A 24-week, randomized, double-blind study of donepezil in moderate to severe Alzheimer's disease. Neurology. 2001;57:613-20.

4. Winblad B, Kilander L, Eriksson S, Minthon L, Båtsman S, Wetterholm AL, et al. Donepezil in patients with severe Alzheimer's disease: doubleblind, parallel-group, placebo-controlled study. Lancet. 2006;367:1057-65.

5. Tan C-C, Yu J-T, Wang H-F, Tan M-S, Meng X-F, Wang C, et al. Efficacy and safety of donepezil, galantamine, rivastigmine, and memantine for the treatment of Alzheimer's disease: A systematic review and meta-analysis. J Alzheimers Dis. 2014;41:615-31.

6. Gauthier S, Schlaefke S. Efficacy and tolerability of Ginkgo biloba extract EGb $761{ }^{\circledR}$ in dementia: a systematic review and meta-analysis of randomized placebo-controlled trials. Clin Intervent Aging. 2015; 9:2065-2077.

7. Tan MS, Yu JT, Tan CC, Wang HF, Meng XF, Wang C, Jiang T, Zhu XC, Tan L. Efficacy and adverse effects of Ginkgo biloba for cognitive impairment and dementia: A systematic review and meta-analysis: J Alzheimers Dis. 2015;43:589-603. 
8. Yang M, Xu DD, Zhang Y, Liu X, Hoeven R, Cho WC. A systematic review on natural medicines for the prevention and treatment of Alzheimer's disease with meta-analyses of intervention effect of ginkgo: Am J Chin Med. 2014;42:505-521.

9. Busse R, Garrido MV, Zentner A, Gericke C. Ginkgohaltige Präparate bei Alzheimer Demenz [Internet]. IQWiG-Bericht. 2008, p. IV. Available from: https://www.iqwig.de/download/A0519B_Abschlussbericht_Ginkgohaltige Praeparate_bei_Alzheimer_Demenz.pdf

10. Yancheva S, Ihl R, Nikolova G, Panayotov $P$, Schlaefke S, Hoerr R. Ginkgo biloba extract EGb 761(R), donepezil or both combined in the treatment of Alzheimer's disease with neuropsychiatric features: a randomised, doubleblind, exploratory trial. Aging Ment Health. 2009;13(2):183-90.

11. Canevelli M, Adali N, Kelaiditi E, Cantet C, Ousset PJ, Cesari M. Effects of Gingko biloba supplementation in Alzheimer's disease patients receiving cholinesterase inhibitors: Data from the ICTUS study. Phytomedicine. 2014; 21:888-892.

12. Kehr J, Yoshitake S, Ijiri S, Koch E, Nöldner M, Yoshitake T. Ginkgo biloba leaf extract (EGb 761) and its specific acylated flavonol constituents increase dopamine and acetylcholine levels in the rat medial prefrontal cortex: possible implications for the cognitive enhancing properties of EGb 761 . Int Psychogeriatr. 2012;24 Suppl 1:S25-34.

13. Lang D, Ude C, Wurglics M, Schubert-Zsilavecs M, Klein J. Brain permeability of bilobalide as probed by microdialysis before and after middle cerebral artery occlusion in mice. J. Pharm. Pharmaceut. Sci. 2010;13:607-614.

14. Ude C, Paulke A, Nöldner M, Schubert-Zsilavecs M, Wurglics M. Plasma and brain levels of terpene trilactones in rats after an oral single dose of standardized Ginkgo biloba extract EGb 761®. Planta medica. 2011;77(3):259-64.

15. Kosasa T, Kuriya Y, Matsui K, Yamanishi Y. Inhibitory effects of donepezil hydrochloride (E2020) on cholinesterase activity in brain and peripheral tissues of young and aged rats. Eur $\mathbf{J}$ Pharmacol. 1999;386(1):7-13.

16. Erb C, Troost J, Kopf S, Schmitt U, Löffelholz K, Soreq H, Klein J. Compensatory mechanisms enhance hippocampal acetylcholine release in transgenic mice expressing human acetylcholinesterase. J Neurochem. 2001;77:63846.

17. Kopf SR, Buchholzer ML, Hilgert M, Löffelholz K, Klein J. Glucose plus choline improve passive avoidance behaviour and increase hippocampal acetylcholine release in mice. Neuroscience. 2001;103(2):365-71.

18. Paxinos G, Watson C. The Rat Brain in Stereotaxic Coordinates. Academic Press. 1998.

19. Hartmann J, Kiewert C, Klein J. Neurotransmitters and energy metabolites in amyloid-bearing
APP $_{\text {SWEXPSEN1dE9 Mouse Brain. J Pharmacol }}$ Exp Ther. 2010;332:364-70.

20. Ellman GL, Courtney KD, Andres V, FeatherStone RM. A new and rapid colorimetric determination of acetylcholinesterase activity. Biochem Pharmacol. 1961;7:88-95.

21. Härtl R, Gleinich A, Zimmermann M. Dramatic increase in readthrough acetylcholinesterase in a cellular model of oxidative stress. J Neurochem. 2011;116(6):1088-96.

22. Zimmermann M, Westwell MS, Greenfield S a. Impact of detergents on the activity of acetylcholinesterase and on the effectiveness of its inhibitors. Biol Chem. 2009;390:19-26.

23. Fonnum F. Radiochemical micro assays for the determination of choline acetyltransferase and acetylcholinesterase activities. Biochem J. 1969;115(3):465-72.

24. Black SA, Ribeiro FM, Ferguson SSG, Rylett RJ. Rapid, transient effects of the protein kinase $\mathrm{C}$ activator phorbol 12-myristate 13-acetate on activity and trafficking of the rat high-affinity choline transporter. Neuroscience. 2010;167(3):765-73.

25. Simon JR, Atweh S, Kuhar MJ. Sodium-dependent high affinity choline uptake: a regulatory step in the synthesis of acetylcholine. J Neurochem. 1976;26(5):909-22.

26. Klein J. Membrane breakdown in acute and chronic neurodegeneration: Focus on choline-containing phospholipids. J Neural Transm. 2000;107:102763.

27. Chang Q, Savage LM, Gold PE. Microdialysis measures of functional increases in ACh release in the hippocampus with and without inclusion of acetylcholinesterase inhibitors in the perfusate. J Neurochem. 2006;97(3):697-706.

28. Thiel CM, Huston JP, Schwarting RKW. Hippocampal acetylcholine and habituation learning. Neuroscience. 1998;85:1253-62.

29. Giovannini MG, Rakovska A, Benton RS, Pazzagli M, Bianchi L, Pepeu G. Effects of novelty and habituation on acetylcholine, GABA, and glutamate release from the frontal cortex and hippocampus of freely moving rats. Neuroscience. 2001;106:43-53.

30. Napoleone P, Ferrante F, Ghirardi O, Ramacci MT, Amenta F. Age-dependent nerve cell loss in the brain of Sprague-Dawley rats: effect of long term acetyl-L-carnitine treatment. Arch Gerontol Geriatr. 10(2):173-85.

31. Nunzi MG, Milan F, Guidolin D, Toffano G. Dendritic spine loss in hippocampus of aged rats. Effect of brain phosphatidylserine administration. Neurobiol Aging. 8(6):501-10.

32. Goh CW, Aw CC, Lee JH, Chen CP, Browne ER. Pharmacokinetic and pharmacodynamic properties of cholinesterase inhibitors donepezil, tacrine, and galantamine in aged and young hooded rats. Drug Metab Dispos. 2011;39:402-11. 
33. Black SA, Rylett RJ. Choline transporter CHT regulation and function in cholinergic neurons. Cent Nerv Syst Agents Med Chem. 2012;12(2):114-21.

34. Tomassoni D, Catalani A, Cinque C, Di Tullio MA, Tayebati SK, Cadoni A, et al. Effects of cholinergic enhancing drugs on cholinergic transporters in the brain and peripheral blood lymphocytes of spontaneously hypertensive rats. Curr Alzheimer Res. 2012;9(1):120-7.

35. DeFeudis F V, Drieu K. Ginkgo biloba extract (EGb 761) and CNS functions: basic studies and clinical applications. Curr Drug Targets. 2000;1:25-58.

36. Stackman R, Eckenstein F, Frei B, Kulhanek D, Nowlin J, Quinn J. Prevention of age-related spatial memory deficits in a transgenic mouse model of Alzheimer's disease by chronic Ginkgo biloba treatment. Exp Neurol. 2003;184:510-20.

37. Wang Y, Wang L, Wu J, Cai J. The in vivo synaptic plasticity mechanism of EGb 761-induced enhancement of spatial learning and memory in aged rats. Br J Pharmacol. 2006; 148:147-53.

38. Blecharz-Klin K, Piechal A, Joniec I, Pyrzanowska J, Widy-Tyszkiewicz E. Pharmacological and biochemical effects of Ginkgo biloba extract on learning, memory consolidation and motor activity in old rats. Acta Neurobiol Exp (Wars). 2009;69:217-31.
39. Yoshitake T, Yoshitake S, Kehr J. The Ginkgo biloba extract EGb 761(R) and its main constituent flavonoids and ginkgolides increase extracellular dopamine levels in the rat prefrontal cortex. $\mathrm{Br} \mathrm{J}$ Pharmacol. 2010 Feb;159(3):659-68.

40. Mdzinarishvili A, Sumbria R, Lang D, Klein J. Ginkgo extract EGb761 confers neuroprotection by reduction of glutamate release in ischemic brain. J. Pharm. Pharmaceut. Sci. 2012;15:94-102.

41. Klein J, Chatterjee S S, and Löffelholz K. Phospholipid breakdown and choline release under hypoxic conditions: inhibition by bilobalide, a constituent of Ginkgo biloba. Brain Res. 1997;755:347-350.42. T. Schwarzkopf, K. Koch and J. Klein (2013) Neurodegeneration after transient brain ischemia in aged mice: beneficial effects of bilobalide. Brain Res. 1529: 178-187.

43. Bierer LM, Haroutunian V, Gabriel S, Knott PJ, Carlin LS, Purohit DP, et al. Neurochemical correlates of dementia severity in Alzheimer's disease: relative importance of the cholinergic deficits. J Neurochem. 1995;64:749-60.

44. Schurr A. Lactate: the ultimate cerebral oxidative energy substrate? J Cereb Blood Flow Metab. 2006;26:142-52.

45. Pellerin L, Magistretti PJ. Neuroenergetics: calling upon astrocytes to satisfy hungry neurons. Neuroscientist. 2004;10:53-62. 